\title{
Extradural Peripheral Nerve Sheath Tumour at T7 Level in a 2-Year-Old Dog
}

\author{
Federica Aragosa ${ }^{1}$ Chiara Caterino ${ }^{1}$ Giovanni Della Valle ${ }^{1}$ Ilaria D'Aquino ${ }^{1}$ Dario Costanza ${ }^{2}$ \\ Carlo Cantile $^{3}$ Gerardo Fatone ${ }^{10}$
}

${ }^{1}$ Department of Veterinary Medicine and Animal Production, University of Naples "Federico II," Naples, Italy

2 Interdepartmental Center of Veterinary Radiology, University of Napoli “Federico II," Naples, Italy

${ }^{3}$ Department of Veterinary Science, University of Pisa, Pisa, Italy

VCOT Open 2021;4:e41-e46.
Address for correspondence Giovanni Della Valle, DMV, PhD, Department of Veterinary Medicine and Animal Production, University of Naples "Federico II," Naples 80137, Italy (e-mail: giovanni.dellavalle@unina.it).

\begin{abstract}
Keywords

- thoracolumbar neoplasia

- extradural tumours

- nerve sheath tumour

- spine

- canine

The aim of this report is to describe an unusual localization of nerve sheath tumour (NST), clinical presentation, imaging, surgical management, and outcome in a 2-yearold dog. A 2-year-old female American Staffordshire Terrier presented with nonambulatory paraparesis, thoracolumbar hyperaesthesia, hindlimb hyperreflexia, and mild muscle atrophy. Computed tomography and magnetic resonance imaging revealed an extradural mass at T7-T8, without vertebral lesions. Surgical treatment consisted in resection of the soft tissue mass through dorsal laminectomy. The dog was ambulatory within 24 hours and free of recurrence at 18 months postoperatively. Histopathologic and features of immunohistochemistry were consistent with NST. The NST of this report was similar to those described before, but exhibited unusual characteristics, such as being extradural, without extension into intervertebral foramina, and being located in an atypical region (T7-T8). Moreover, survival time and relapsefree interval are greater than previously reported for similar cases.
\end{abstract}

\section{Introduction}

Intraspinal neoplasms can involve the spinal cord, dura mater, spinal nerves, or paraspinal tissues, resulting in clinical signs of spinal dysfunction. According to their location relative to spinal cord and dura mater, they are classified as intramedullary (IM), intradural-extramedullary (ID-EM), or extradural (ED). ${ }^{1,2}$ The ED tumours have been described to account for $50 \%$ of all spinal cord neoplasms in dogs, followed by ID-EM reported with an overall distribution of 35\%; IM tumours are least common, comprising the remaining $15 \%{ }^{2}$

Peripheral nerve sheath tumours (PNSTs) are a heterogeneous group of soft tissue neoplasms, both benign and malignant, arising from Schwann cells or modified Schwann cells, endoneurial fibroblasts, or perineural cells. ${ }^{3}$ The latest

received

January 13, 2021

accepted after revision

May 2, 2021
2007 World Health Organization (WHO) classification of human PNSTs was adapted to veterinary medicine due to the remarkable gross and histological similarities of these tumours in people and dogs. ${ }^{4}$ According to this classification, there are four major subtypes: schwannoma, neurofibroma, perineurioma, and malignant peripheral nerve sheath tumour (MPNST). ${ }^{4}$ Any part of a nerve or nerve root can be affected, involving spinal nerve roots in the caudal cervical and cranial thoracic region, nerves contributing to the brachial plexus, ${ }^{3,5-7}$ as well as cranial nerves and other peripheral nerves. ${ }^{3,8-10}$ One report describes schwannoma as being the second most common spinal tumour in dogs after meningioma. ${ }^{11}$ However, compared with other spinal cord affections, such as trauma and intervertebral disc disease, neoplasms are the least common compressive lesion., ${ }^{72}$

(c) 2021. The Author(s).

This is an open access article published by Thieme under the terms of the Creative Commons Attribution License, permitting unrestricted use, distribution, and reproduction so long as the original work is properly cited. (https://creativecommons.org/licenses/by/4.0/)

Georg Thieme Verlag KG, Rüdigerstraße 14, 70469 Stuttgart, Germany 

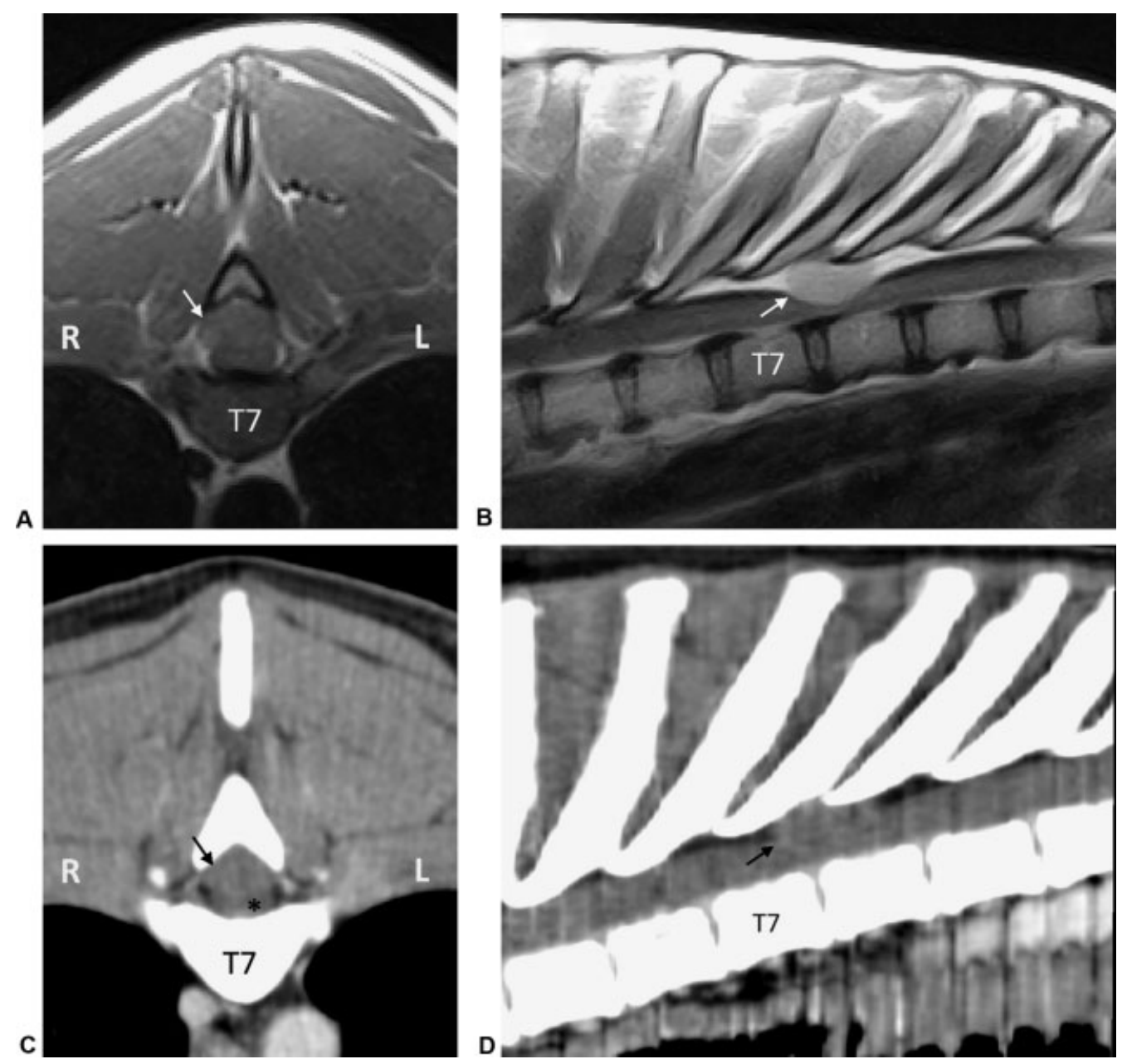

Fig. 1 Transverse and sagittal magnetic resonance imaging (MRI) T1-weighted (A, B) and contrast-enhanced computed tomography (CT) (C, D) images of the spina at the level of the caudal end of T7 body. A mass (arrows) is visible inside the vertebral canal and compressing the underlying spinal cord (asterisk). The mass appears to be hyperintense in T1-weighted MRIs (A, B) and moderately enhancing in CT images (C, D). L, left; R, right.

Current treatment options for spinal tumours include surgical resection, ${ }^{5,6}$ stereotactic radiation therapy (SRT), ${ }^{1,13,14}$ and, occasionally, chemotherapy. ${ }^{15}$ The PNSTs have a high rate of recurrence, and the overall prognosis is considered poor, depending on the completeness of local resection, spinal cord infiltration and collateral damage before and during surgery, and on tumour type.,

Herein we report a PNST in a 2-year-old dog, extradurally at T7, which is an infrequent location, ${ }^{1,3,5}$ and the successful long-term outcome after surgical excision.

\section{Case Description}

A 2-year-old, intact female American Staffordshire Terrier, weighing $26 \mathrm{~kg}$, presented with a 1-month history of hindlimb paraplegia, with no known history of trauma. The dog underwent previously treatment with prednisone $(1 \mathrm{mg} / \mathrm{kg} \mathrm{q} .12$ per os [PO], Prednicortone, Dechra Veterinary Products S.r.l., Turin, Italy) which temporarily yielded some improvement. At referral to the reporting institution, the dog presented with non-ambulatory paraparesis, hypertonic hindlimb muscles, lack of proprioception, and hyperreflexia of the hindlimbs (patellar and cranial tibial reflex). Deep palpation of the thoracolumbar vertebral region elicited pain in the T5-T10 area, and the cutaneous trunci reflex was lost caudal to T10. Complete blood count, serum biochemical examinations, and cardiologic evaluation were unremarkable.

Magnetic resonance imaging (MRI) and computed tomography scan (CT) were performed 20 days before being referred to the teaching hospital ( $\mathbf{F i g}$. 1). The CT scan showed a well-defined ED mass $(11 \times 10 \times 18 \mathrm{~mm})$, dorsally to the spinal cord at the level of T7-T8, causing moderate compression of the dural sac. Contrast-enhanced CT images confirmed the absence of other alterations affecting the vertebral column. On MRI, the mass was hyperintense on T1-weighted and short tau inversion recovery sequences and hypointense on T2-weighted images; mild to moderate heterogeneous contrast enhancement was present, not extending outside the vertebral canal, with normal profiles of vertebral bodies and intervertebral foramina. There were no signs attributable to lung or lymph node metastasis, neither on CT nor on MRI.

The imaging features were most compatible with meningioma, fibrosarcoma, and PNST, but hemangiosarcoma, 

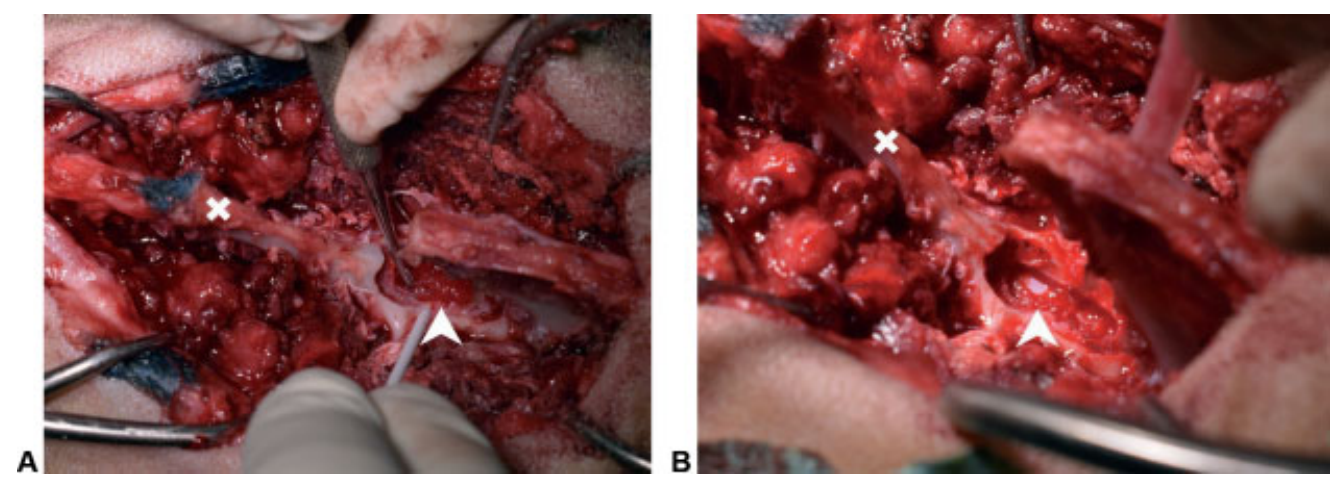

Fig. 2 Intraspinal extradural mass (white arrowhead) at the T7 level during (A) and after removal (B) using a T7-T8 dorsal laminectomy. White X: T6 spinal process.

lymphosarcoma, plasmacytoma, or inflammatory granuloma could not be ruled out. ${ }^{1,6,16}$ Surgical exploration, attempting to excise the lesion, was proposed and executed as follows: after routine premedication and in general anaesthesia, the dog was positioned in sternal recumbency. Anaesthesia was maintained with isoflurane (Isoflo, Ecuphar Italia S.r.l., Milan, Italy) in oxygen and fentanyl (Fentadon, Dechra Veterinary Products S.r.l., Turin, Italy) in constant rate infusion.

A dorsal T7-T8 laminectomy was performed, ${ }^{17}$ involving the removal of the spinous process of $\mathrm{T} 7$, the dorsal lamina, and caudal articular processes, while cranial articular processes were preserved ( $\mathbf{- F i g . 2}$ ). After sufficient exposure of the vertebral canal, a reddish-grey soft tissue mass was found. The mass had a smooth surface and was loosely attached to the dura mater with thin connective tissue strands. The spinal cord, as far as visible without durotomy, had a normal appearance, and no haemorrhages were seen. By gentle blunt dissection, the mass could be excised entirely, avoiding damage to the dura mater; no unusual bleeding was encountered, but preventively, a gelatine sponge (GELITASPON, Gelita Medical) was placed on the spinal cord. To manage any residual vertebral instability, a figure-eight cerclage wire $(\varnothing 1.2 \mathrm{~mm}$, Alcyon Italia S.P.A.) was placed between the spinous processes of T6 and T8. Reconstruction of paraspinal musculature, fascia, subcutaneous tissues, and the skin was made in routine fashion.

Postoperative care consisted of carprofen $(4 \mathrm{mg} / \mathrm{kg}$ q24h SC, Rimadyl, Zoetis Italia S.r.l., Rome, Italy) and ceftriaxone (25 mg/kg q12h PO, Mylan Italia S.r.l., Milan, Italy) medication. The patient was ambulatory within 24 hours after surgery and was discharged after 3 days, exhibiting only mild ataxia.

For histologic examination, the excised tissue was routinely processed for paraffin sections $(4 \mu \mathrm{m})$ and stained with haematoxylin and eosin, Masson's trichrome, and Van Gieson. Histopathological examination revealed a hypercellular, unencapsulated mass, featuring a population of densely packed spindle-shaped cells, characterized by thin and eosinophilic cytoplasm, atypical plump, ovoid, single, or multiple nuclei, and prominent nucleoli (-Fig. 3). The predominant pattern consisted of areas of interwoven bundles, streams, or concentric whorls. Moderate cellular pleomorphism and anisokaryosis were observed, but mitotic figures were infrequent $(\sim 0-1$ in 10 fields at high power magnification). Infiltrate characterized by lymphocytes, plasma cells, histiocytoid cells, and rare macrophages was noted. Van Gieson and Masson's trichrome stain showed scattered stroma and fine delicate collagen fibres in few areas of the tumour. For further tumour typing, immunochemistry was performed using a polyclonal antibody against laminin, S-100 protein, vimentin, and glial fibrillar acid protein (GFAP). Immunohistochemistry revealed that the neoplasm was immunoreactive for vimentin, GFAP, and S100 protein. Furthermore, we performed immunohistochemistry using an anti-laminin antibody, and did not observe immunopositivity for this antibody. Immunoreactivity against those markers was consistent with a benign PNST.

At 1 week after surgery, there was complete remission of neurological signs, and at 1,3,6, and 9 months follow-up, the absence of any neurologic deficits confirmed full recovery. However, a 13-month follow-up by CT scan (-Figs. 4-5) revealed in the identical localization a small, round-shaped ED lesion within the vertebral canal, moderately enhancing by contrast medium. No compression on the dural sac and no further alterations of the spinal cord and vertebral metamere were noted. These imaging findings were suggestive of scar tissue resulting from surgery, forasmuch as no neurological signs recurred.

At the time of writing, 18 months after surgery, the dog is free of any neurological and/or clinical signs.

\section{Discussion}

The PNSTs are a heterogeneous group of neoplasms that may originate from Schwann cells or related cells of the perineural sheath (schwannomas and perineuriomas) or from a combination of endoneurial, epineurial, or stromal fibroblasts (neurofibromas and MPNSTs)., ${ }^{3,7}$ Difficulties in histologic differentiation and the overlapping biological behaviour summarize these tumours in the general definition of PNSTs. ${ }^{3,18}$

Peripheral nerve sheath tumours develop mainly from peripheral nerves, but they may extend along the nerve roots through the intervertebral foramen developing an EM component or may even involve the spinal cord's grey matter (e.g. as dumbbell tumours)., ${ }^{3,19,20}$ Nevertheless, 
A
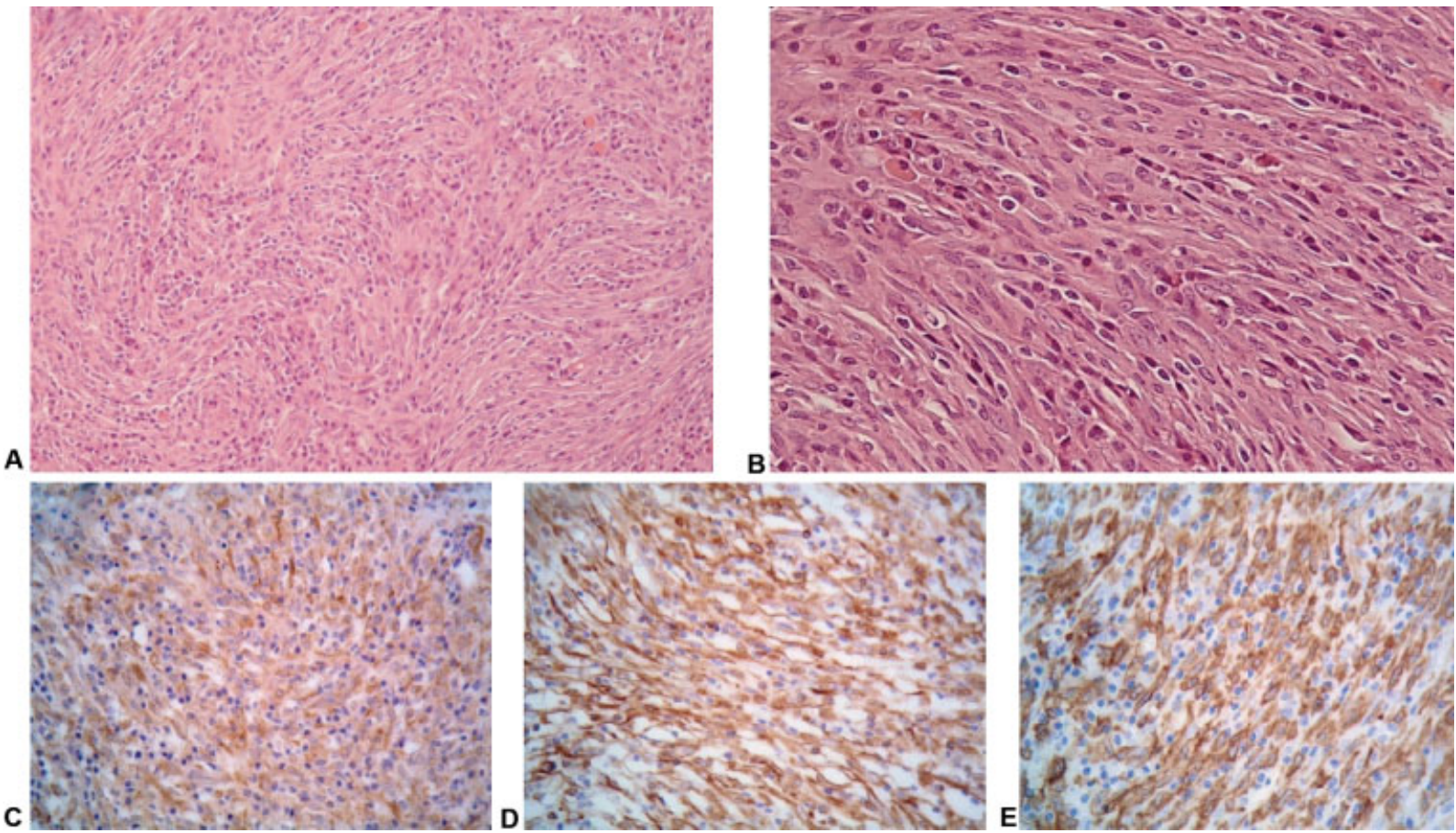

Fig. 3 Histopathological examination of the resected thoracic mass in $\times 20$ magnification (A) and $\times 40$ magnification (B). The tumour is composed of a cell population organized in densely packed spindle-shaped cells. The predominant pattern consisted of areas of interwoven bundles, streams or concentric whorls. Tumour cells present uniformly intense immunopositivity for vimentin protein (C), S100 protein (D) and glial fibrillar acid protein $(\mathrm{E})(\times 40)$.

primary intraspinal-extradural PNSTs have been reported in dogs. ${ }^{1,6,20}$ In people, tumours with an extraforaminal component seem to decrease in frequency from the upper to the lower region of the spine. An explanation could be relatable to the anatomic features of nerve roots that arise from the spinal cord at a nearly right angle in the cervical vertebrae and at more acute angles in the lower spinal tract, running longer distances within the vertebral canal to reach the intervertebral foramina. ${ }^{21}$ To the authors' knowledge, this is the first description in the dog of an ED PNST with well-defined margins, located at T7-T8 level, and without any involvement of osseous vertebral structures and inter- vertebral foramina. Also, as previously reported, the median age of dogs with PNSTs was 7.7 years. ${ }^{1,7}$ Therefore, our 2year-old patient was relatively young compared with previous reports. Neither gender nor breed predilection has been demonstrated. ${ }^{1,5}$ Clinical signs are mainly related to the anatomical location and progressive spinal cord compression, consistent with the clinical presentation of our case. $^{6}$

The PNSTs are slow-growing, locally invasive and have a low rate of metastasis. ${ }^{1}$ Nevertheless, a recent study on canine soft-tissue sarcomas reported an $11.1 \%$ (27/242 cases) frequency of metastasis of PNST. ${ }^{22}$ In general, lungs and
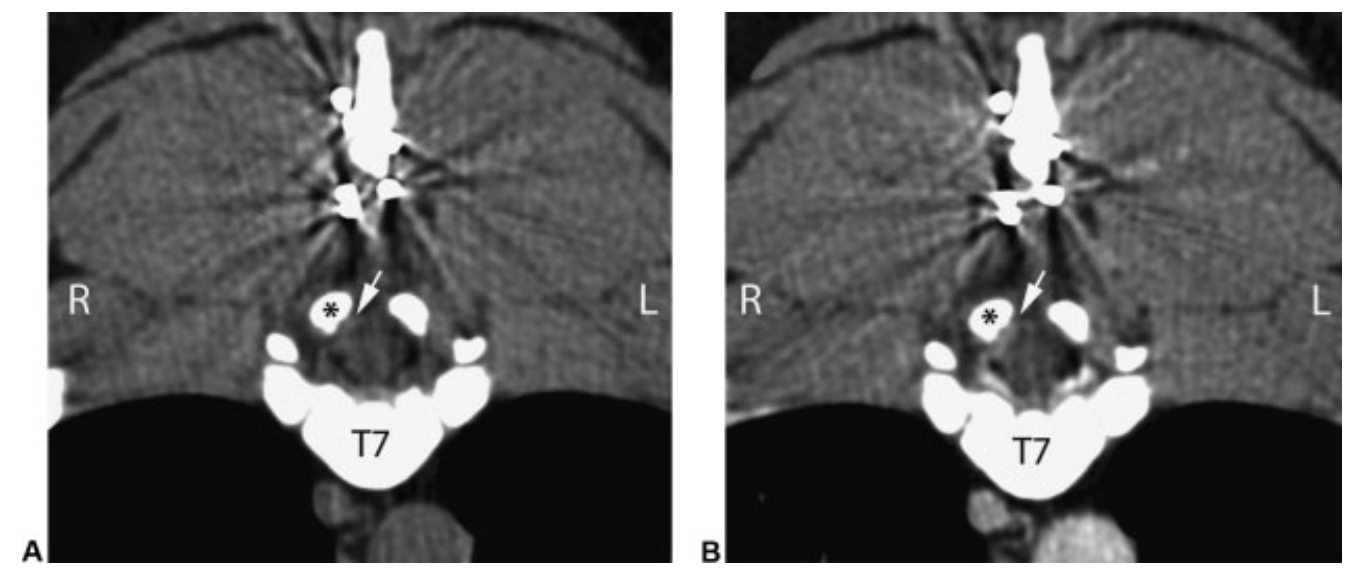

Fig. 4 Transverse computed tomographic images, obtained 13 months after surgery, at the level of the caudal end of the T7 body, before (A) and after (B) the contrast-medium administration (WW: 360, WL: 60). In the vertebral canal, under the T7 right caudal articular process (asterisk) a small lesion with slight contrast enhancement (arrow) is visible. L, left; R, right. 

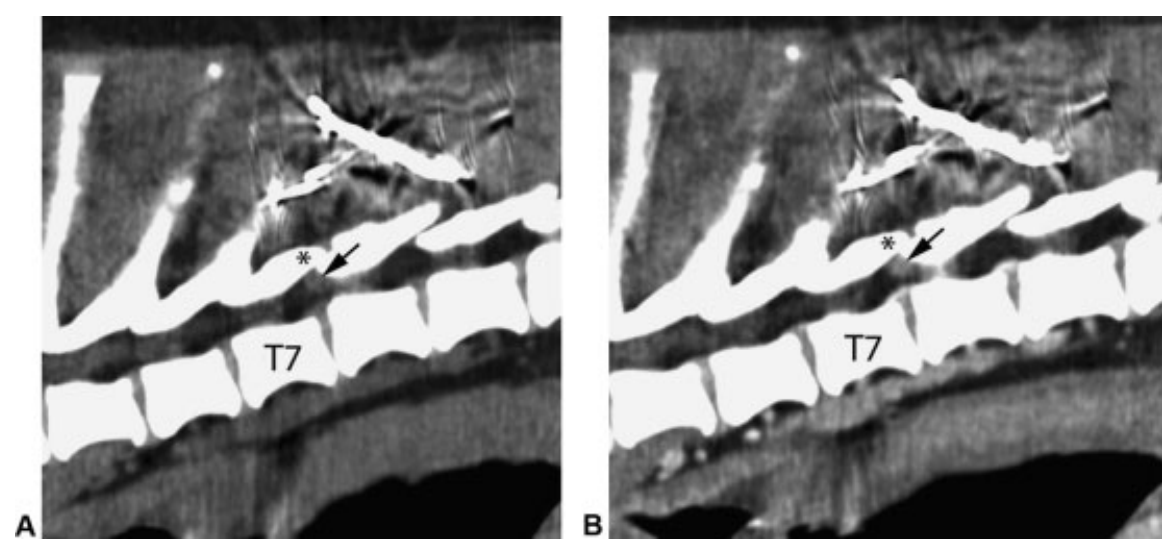

Fig. 5 Right sagittal multiplanar reconstructed images of the thoracic spine (from T5 to T9), before (A) and after (B) the contrast-medium administration (WW: 360, WL: 60), obtained 13 months after surgery. In the vertebral canal, under the T7 caudal articular process (asterisk), a small, rounded lesion with a slight contrast enhancement (arrow) is visible.

regional lymph nodes are the most common site for metastasis of soft tissue sarcomas ${ }^{23}$; there was no metastasis detectable in the present case. As for all neoplasms, early recognition is essential in PNSTs as well. Diagnosis often requires advanced imaging techniques, ${ }^{16,24}$ such as $\mathrm{CT}$ and MRI. The PNST of this report had on MRI an appearance similar to cases described before but showed some unusual characteristics, such as being ED and located in the midthoracic spinal region, entirely within the vertebral canal.

Complete surgical excision of PNSTs is associated with significantly improved survival in both animal and human patients. ${ }^{1,5,6}$ The final prognosis depends upon the degree of local resection, degree of spinal infiltration, direct damage to the spinal cord by the lesion itself or during surgical manipulation, surgeon's experience, and tumour type. ${ }^{6}$ Extradural tumours are removed by laminectomy, either dorsal or by hemilaminectomy, via blunt or sharp dissection. General surgical oncologic principles, like wide surgical margins, are often not feasible in neurosurgical oncology because of the obvious vital nature of the surrounding nervous system. ${ }^{1,5}$ The 'en bloc' resection reduces the risk of local recurrence and results in prolonged survival; on the contrary, incomplete resection resulted in tumour recurrence and fatal outcome. ${ }^{9}$ Local excision was performed, but wide surgical margins obviously were not achievable in our case.

The beneficial effect of SRT in terms of survival is reported, ${ }^{1,10,13}$ despite additional survival time was noted only adding SRT when recurrence was noted. ${ }^{1,25}$ Therefore, surgery remains the treatment of choice. ${ }^{1}$ Despite aggressive and timely management, the overall prognosis is considered poor for most cases of PNSTs. In several cases, dogs were euthanatized because of worsening of clinical signs or in instances because of owner's decision not to treat. $3,5,7,20,26$ Furthermore, survival time for PNSTs managed surgically ranged from 10 days to 18 months with a median survival time of 5 months, ${ }^{5}$ and in a recent retrospective multicentre case series, median survival time was 187 days (6.2 months). ${ }^{1}$ However, comparison of survival time after surgery is difficult because in these reports, several cases of recurrence were treated with SRT. ${ }^{1}$ Nevertheless, these reports are similar to what was previously described for 12 cases of spinal PNSTs with a median survival time of 180 days. ${ }^{27}$ Conversely, dogs that underwent hemilaminectomy with rhizotomy for PNST survived for only 2 or 3 months, according to another recent report. ${ }^{6}$ Inconsistent data exist on recurrence of spinal PNST. One study reported a median relapse-free interval of 108 days postoperatively in 7 of 13 dogs treated with surgery alone. ${ }^{1}$ Another study on 23 dogs showed recurrence at 1 month (0-14 months $)^{5}$; conversely, recurrence was noted at 7 months after surgery in two recent case reports. ${ }^{14,19}$ Our patient did not show recurrence of clinical signs within 18 months (540 days) after treatment, and the absence of local recurrence seemed to be confirmed by the CT scan 13 months (390 days) after surgery.

Macroscopical and histological features of the mass described reflect what was previously reported. ${ }^{6,7,20,24}$ The diagnosis of PNST in our case was confirmed by histopathological examination and immunohistochemical labelling. Immunohistochemistry revealed that the neoplasm was immunoreactive for vimentin, GFAP, and S-100 protein but was immunonegative for anti-laminin antibody. In a previous study, only $33.3 \%$ of PNSTs had positive immunoreactivity against this marker. ${ }^{28}$ The positive result for vimentin confirmed that the tumour was of mesenchymal origin, while meningioma, fibrosarcoma, and lymphosarcoma were excluded by the positive reaction to S-100. Moreover, a positive result for GFAP and the low number of mitotic figures were consistent with benign behaviour. ${ }^{29}$ Fibrous meningiomas may resemble schwannomas, and the whorl formation in transitional meningiomas can mimic that of schwannomas. However, meningiomas are S-100 negative and can be distinguished ultrastructurally by their characteristic interdigitating cellular processes, prominent desmosomal and gap junctions, and lack of a basal lamina. ${ }^{30}$ Although rhabdomyosarcomas and hemangiopericytomas can be positive to S-100, they show only focal immunoreactivity for S-100 and usually involve less than $20 \%$ of the cells. $^{29}$

This case represents, to the best of our knowledge, the first report in a dog with a primary ED PNST in the T7-T8 location; also, the dog was much younger than others with spinal 
PNSTs reported so far, was successfully treated by surgical excision alone and had a relapse-free outcome of at least 18 months.

Peripheral nerve sheath tumours should be considered in the differential diagnosis of dogs showing an intraspinal ED mass without any signs of extension through a neuroforamen on advanced imaging.

\section{Authors' Contribution}

G.F., G.D.V., F.A., and C.C. performed clinical evaluation, surgical procedure, and follow-up assessment. F.A. and C.C. were involved in preparation and revision of the manuscript. D.C. performed imaging. I.D.A. performed histological diagnosis; Ca.Ca performed immunochemistry. G.F., G.D.V., D.C., and I.D.A. contributed to the final revision of the manuscript. All authors gave their final approval of the manuscript.

\section{Conflict of Interest}

Nothing to declare.

\section{References}

1 Lacassagne K, Hearon K, Berg J, et al. Canine spinal meningiomas and nerve sheath tumours in 34 dogs (2008-2016): distribution and long-term outcome based upon histopathology and treatment modality. Vet Comp Oncol 2018;16(03):344-351

2 Pancotto TE, Rossmeisl JH Jr, Zimmerman K, Robertson JL, Werre SR. Intramedullary spinal cord neoplasia in 53 dogs (1990-2010): distribution, clinicopathologic characteristics, and clinical behavior. J Vet Intern Med 2013;27(06):1500-1508

3 Jones BR, Alley MR, Johnstone AC, Jones JM, Cahill JI, McPherson C. Nerve sheath tumours in the dog and cat. N Z Vet J 1995;43(05): 190-196

4 Louis DN, Ohgaki H, Wiestler OD, et al. The 2007 WHO classification of tumours of the central nervous system. Acta Neuropathol 2007;114(02):97-109

5 Brehm DM, Vite CH, Steinberg HS, Haviland J, van Winkle T. A retrospective evaluation of 51 cases of peripheral nerve sheath tumors in the dog. J Am Anim Hosp Assoc 1995;31(04):349-359

6 Besalti O, Caliskan M, Can P, Vural SA, Algin O, Ahlat O. Imaging and surgical outcomes of spinal tumors in 18 dogs and one cat. J Vet Sci 2016;17(02):225-234

7 Chaigneau FC, Maldonado EB, Casas FC, Mejía CS, Ramírez LIM, Ruiz CG. Peripheral nerve sheath tumor in the cervical area of the vertebral canal in a dog. Vet Mex 2007;38(02):241-247

8 Green M, Pumarola M, Targett M, vanLelyveld S, Lowrie M. Malignant trigeminal nerve Schwannoma diagnosed via biopsy of the infraorbital nerve in a dog. Vet Rec Case Rep 2020;8: e001057

9 van Stee L, Boston S, Teske E, Meij B. Compartmental resection of peripheral nerve tumours with limb preservation in 16 dogs (1995-2011). Vet J 2017;226:40-45

10 Swift KE, McGrath S, Nolan MW, et al. Clinical and imaging findings, treatments, and outcomes in 27 dogs with imaging diagnosed trigeminal nerve sheath tumors: a multi-center study. Vet Radiol Ultrasound 2017;58(06):679-689

11 Wright JA, Bell DA, Clayton-Jones DG. The clinical and radiological features associated with spinal tumours in thirty dogs. J Small Anim Pract 1979;20(08):461-472
12 Fingeroth JM, Prata RG, Patnaik AK. Spinal meningiomas in dogs: 13 cases (1972-1987). J Am Vet Med Assoc 1987;191(06):720-726

13 Hansen KS, Zwingenberger AL, Théon AP, Pfeiffer I, Kent MS. Treatment of MRI-diagnosed trigeminal peripheral nerve sheath tumors by stereotactic radiotherapy in dogs. J Vet Intern Med 2016;30(04):1112-1120

14 Narita M, Nishida H, Goto S, et al. Primary malignant peripheral nerve sheath tumors arising from the spinal canal invading the abdominal cavity in a dog. J Vet Med Sci 2020;82(04):452-456

15 SonJ, ParkS, Seok HC, Kim G. Treatment of malignant peripheral nerve sheath tumor using surgery and metronomic chemotherapy in a dog.J Vet Clin 2011;3:310-313 Last accessed June 9, 2021 at: https://www. koreascience.or.kr/article/JAKO201122234006605.page

16 Kippenes H, Gavin PR, Bagley RS, Silver GM, Tucker RL, Sande RD. Magnetic resonance imaging features of tumors of the spine and spinal cord in dogs. Vet Radiol Ultrasound 1999;40(06):627-633

17 Fisher C, Shores A. Dorsal laminectomy in the thoracolumbar region. In: Shores A, Brisson BA, eds. Current Techniques in Canine and Feline Neurosurgery. 1st edition. Hoboken, NJ: Wiley Blackwell; 2017:205-207

18 Suzuki S, Uchida K, Nakayama H. The effects of tumor location on diagnostic criteria for canine malignant peripheral nerve sheath tumors (MPNSTs) and the markers for distinction between canine MPNSTs and canine perivascular wall tumors. Vet Pathol 2014;51 (04):722-736

19 Poli F, Calistri M, Mandara MT, Baroni M. Central nervous system metastasis of an intradural malignant peripheral nerve sheath tumor in a dog. Open Vet J 2019;9(01):49-53

20 Oliveira M, De La Fuente C, Pumarola M, Añor S. Imaging diagnosis: cranial cervical intraspinal schwannoma in a dog. Vet Radiol Ultrasound 2014;55(03):300-304

21 Jinnai T, Koyama T. Clinical characteristics of spinal nerve sheath tumors: analysis of 149 cases. Neurosurgery 2005;56(03):510-515, discussion 510-515

22 Bray JP, Polton GA, McSporran KD, Bridges J, Whitbread TM. Canine soft tissue sarcoma managed in first opinion practice: outcome in 350 cases. Vet Surg 2014;43(07):774-782

23 Bray JP. Soft tissue sarcoma in the dog - part 1: a current review. J Small Anim Pract 2016;57(10):510-519

24 Drost WT, Love NE, Berry CR. Comparison of radiography, myelography and computed tomography for the evaluation of canine vertebral and spinal cord tumors in sixteen dogs. Vet Radiol Ultrasound 1996;37(01):28-33. Doi: 10.1111/j.1740-8261.1996. tb00808.x

25 Lourinho F, Trevail R, Craig A, Carrera I. Presumptive malignant nerve sheath tumour affecting the cauda equina and caudal nerves of a dog. Vet Rec Case Rep 2018;6:e000626-10. Doi: 1136/vetreccr-2018-000626

26 Uchida K, Nakayama H, Sasaki N, Tateyama S, Goto N. Malignant schwannoma in the spinal root of a dog. J Vet Med Sci 1992;54(04): 809-811

27 Levy MS, Kapatkin AS, Patnaik AK, Mauldin GN, Mauldin GE. Spinal tumors in 37 dogs: clinical outcome and long-term survival (1987-1994). J Am Anim Hosp Assoc 1997;33(04):307-312

28 Gaitero L, Añor S, Fondevila D, Pumarola M. Canine cutaneous spindle cell tumours with features of peripheral nerve sheath tumours: a histopathological and immunohistochemical study. J Comp Pathol 2008;139(01):16-23

29 Chijiwa K, Uchida K, Tateyama S. Immunohistochemical evaluation of canine peripheral nerve sheath tumors and other soft tissue sarcomas. Vet Pathol 2004;41(04):307-318

30 Higgins RJ, Bollen AW, Dickinson PJ, Sisó-Llonch S. Tumors of the nervous system. In: Tumors in Domestic Animals. 5th edition. Ames, IA: John Wiley \& Sons, Inc.; 2016:834-891 